\title{
IAMJ
}

INTERNATIONAL

AYURVEDIC

MEDICAL JOURNAL

\section{CLINICAL ASSESSMENT OF BASTI KARMA AND RASARAJ RASA IN THE MANAGEMENT OF GRIDHRASI (SCIATICA)}

\author{
Mishra Meenu ${ }^{1}$, Shivhare Shwetal ${ }^{2}$, Sharma Vivek ${ }^{3}$ \\ ${ }^{1} 2^{\text {nd }}$ Year PG Scholar, ${ }^{2}$ Reader, ${ }^{3}$ Lecturer \\ Department of Kayachikitsa, Pt. Khushilal Sharma Govt. (Auto.) Ayurveda College and Institute, Bhopal, \\ Madhya Pradesh, India
}

Corresponding Author: mishrameenu654@gmail.com

https://doi.org/10.46607/iamj1808102020

(Published online: October 2020)

Open Access

(C) International Ayurvedic Medical Journal, India 2020

Article Received:26/09/2020 - Peer Reviewed:02/10/2020 - Accepted for Publication:04/10/2020

Check for updates

\section{ABSTRACT}

Background: Sciatica is the most common disorder which affects the quality of life of individual. Sciatica is the set of symptoms which is caused by a pinching and /or irritation of one of the three lowest nerve roots that make up the giant sciatic nerve and causing a low back pain and leg pain. Prevalence of sciatica ranges from $2 \%$ to $40 \%$ worldwide. In Ayurveda sciatica has a high resemblance with Gridhrasi, which comes under Samanyaja Vikara and Nanatmaja Vatavikara. Aim \& Objectives: To study the effect of Dashmooladi Niruha Basti with Rasaraj Rasa in the management of Gridhrasi (Sciatica) and to find out an effective Ayurvedic management for Gridhrasi (Sciatica). Material and Methods: An open clinical study with Dasmooladi Niruha Basti with Rasaraj Rasa was done in 10 patients of Gridhrasi (sciatica). 10 clinically diagnosed patients of Gridhrasi (sciatica), registered at the Kayachikitsa OPD of Pt. Khushilal Sharma Govt. Ayurvedic Hospital Bhopal. The patients were treated with Dasmooladi Niruha Basti along with Rasaraj Rasa. Duration of study is 30 days. Assessment was done on the basis of symptomatic relief and change in the degree of SLR test after treatment. Observation: Dasmooladi Niruha Basti and Rasaraj Rasa yielded symptomatic relief and increased the degree of SLR test after treatment. The overall effect of the treatment in all patients suggested that, complete cure was not in any patient. Three patients were markedly improved, and seven patients were moderately improved. None of patients remained un- 
changed. Conclusion: On the basis of result obtained, it can be concluded that Dasmooladi Niruha Basti and Rasaraj Rasa can be used as an effective treatment in the management of Gridhrasi (sciatica).

Keywords: Gridhrasi, Dasmooladi Niruha Basti, Sciatica, Rasaraj Rasa

\section{INTRODUCTION}

The complexity of the brain differentiates us from other species and its interactions with the spinal cord and peripheral nerves combine to allow us to perceive and react to the external world while maintaining a stable internal environment. The spinal cord and spinal root may be affected by intrinsic disease or by disorders of the surrounding meninges and bones. ${ }^{1}$ Sciatica is caused by impingement of the $\mathrm{L}_{4}, \mathrm{~L}_{5}$ or $\mathrm{S}_{1}$ nerve (i.e. from a herniated disk) and manifests as unilateral neuropathic pain extending from the gluteal region down the posterolateral leg to foot. ${ }^{2}$ Worldwide $2 \%$ to $40 \%$ of people have sciatica. Sciatica has been reported to occur in 1 to $10 \%$ of the population, most commonly in people age 25 to 45 years. Men are more frequently affected than women. The highest incidence is found in the fifth decade and then decreases with increasing age. ${ }^{3}$ Lumber disc herniation is often precipitated by trauma (usually lifting heavy weights while the spine is flexed), genetic factors may also be important. The onset may be sudden or gradual. Alternatively, repeated episodes of low back pain may precede sciatica by months or years. Constant aching pain is felt in the lumber region and may radiate to the buttock, thigh, calf and foot. Pain is exacerbated by coughing or straining but may relieved by lying flat. MRI is the investigation of choice if available, since are of little value in the diagnosis of disc disease, although they may demonstrate conditions affecting the vertebral body. ${ }^{4}$ In Ayurveda sciatica in symptomatology can be correlated with Gridhrasi which comes under Samanyaja Vikara ${ }^{5}$ and Nanatmaja Vatavikara. ${ }^{6}$ It is described in Brihattrayee as well as Laghutrayee. In Gridhrasi, the word Gridhra means a bird called as vulture in English. According to Shabdakalpadruma the word Gridha refers to a person who is crazy of eating meat. Gridhrasi is named so due to resemblance of the abnormal gait of the patient to the gait of bird vulture. Specific causative factors for Gridhra- siare not mentioned in the classics, all the Nidanas which are causing Vatavyadhi are attributed as the etiological factors of Gridhrasi. The vitiation of Vata takes place in two ways viz. Dhatukshaya and Margavarana. ${ }^{7}$ Charak states the Lakshnas of Gridhrasi as the pain starting from Sphik (buttock) and radiating downwards Kati (posterior aspect of waist), Prushta (back region), Uru (posterior aspect of thigh), Janu (knee), Jhangha (calf), and Pada (foot) in order. ${ }^{8}$ SamanayaLakshnas of Gridhrasi are Ruk (pulling pain), Toda (piercing pain), Sthambha (stiffness) and Spandana (twitching) described by Acharya Charak (Ch.Chi.28/56), Madhavakar (Ma.Ni.22/54), Bhavamishra (Madhyamkhanda Vatavyadhi) and Vangsena (Vatavyadhi Adhikara). Prashnipratyanguli Vedana (scoliotic postural deformity) described by Acharya Sushruta (Su.Ni.1/74) and Vaghbatta (A.H.Ni.15 th Chp.Vatavyadhi). Vishishta Lakshanas of Vataja and Vata-Kaphaja Gridhrasi elaborated by Madhavakar (Ma.Ni.22/55-56) and other Acharyas. Modern management of sciatica includes conservative treatment with analgesia and early mobilization. Adjuvant treatment with local anaesthetic or glucocorticoid and surgery may have to be considered if there is no response to conservative treatment. In this research paper a holistic approach is adopted to evaluate the role of Dashmooladi Niruha Basti and Rasaraj Rasa in the management of Gridhrasi. Dashmooladi Niruha Basti as per Ayurvedic guidelines and effect of Rasaraj Ra$s a$ will be observed with respect to its clinical effect in patients.

\section{Aim and Objectives}

1. To study the effect of Dashmooladi Niruha Basti with Rasaraj Rasa in the management of Gridhrasi (Sciatica).

2. To find out an effective Ayurvedic management for Gridhrasi (Sciatica). 


\section{Material and Methods}

Selection and Source of patient: For this open clinical study, 10 patients of Gridhrasi were registered from OPD of Kayachikitsa department and admitted in IPD of Pt. Khushilal Govt. (Auto.) Ayurveda Hospital Bhopal.

Plan of study: Patients taking allopathic medicines were stopped during the study period. The drugs required for Dashmooladi Niruha Basti were procured and prepared in Prakalp of Panchkarma in Pt. KLS Govt. Ayurveda Hospital Bhopal. These registered patients were properly informed regarding the Basti procedure, they would undergo and admitted in the hospital indoor ward and treated under direct supervision. The duration of study: 30 days and the Follow up were followed on completion of trial every week for 2 months.

\section{Samprapti (Pathogenesis)}

Nidana Sevana:

Dhatukshyajanya \& Margavarodha Nidana<smiles>[C]1[C+]=CC=C1</smiles>

Vata or Vatakapha Sanchaya (Sanchaya)

Vataprakopa or Vatakaphaprakopa (Prakopa)

Dushita Vata or Vatakapha enters the Dhamani, Sira, Nadi etc. (Prasara)

?

Dushita Vata or Vatakapha lodged in Rasavaha, Raktavaha \& Mansavaha Srotasa (Shthanasamshraya)

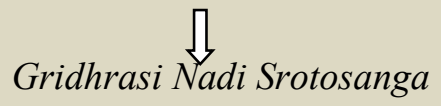

Pain starting from Sphik \& radiating downwards Kati, Prushta, Uru, Janu, Jhangha, and Pada in order etc. symptom complex (Vyaktavastha)<smiles>[CH]=C</smiles>

Manifestation of Gridhrasi

Treatment Regimen: These diagnosed cases of Gridhrasi admitted in indoor ward of Pt. K. L. S.
Govt. Ayurveda Hospital, Bhopal and undergo following procedures

Table 1

\begin{tabular}{|l|l|l|l|l|}
\hline S. No. & Treatment & Drug used & Dose & Duration \\
\hline 1. & $\begin{array}{l}\text { Sthanik Abhyang (Kati region) } \\
\text { and Sthanika Nadi Swedana }\end{array}$ & $\begin{array}{l}\text { Narayan oil \& } \\
\text { Dashmool Siddha Kwatha }\end{array}$ & $\begin{array}{l}\text { Q. S. } \\
\text { Niruha Basti (Dashmooladi Niruha Basti) }\end{array}$ \\
\hline 2. & $\begin{array}{l}\text { Basti plan (Kaal Basti) } \\
\text { Anuvasana Basti (Sahachar Tail) }\end{array}$ & $\begin{array}{l}350 \mathrm{ml} \\
75 \mathrm{ml}\end{array}$ & 16 days \\
\hline 3. & Shaman medicine & Rasaraj Rasa $^{10}$ & $125 \mathrm{mgbd}$ & 30 days \\
\hline
\end{tabular}


Table 2: Ingredient of Dashmooladi Niruha Basti (Ch. Siddhi 3/35)

\begin{tabular}{|l|l|l|}
\hline S. No. & KwathaDravya & Botanical Name \\
\hline $\mathbf{1 .}$ & Bilva & Foeniculum vulgare \\
\hline $\mathbf{2 .}$ & Shyonak & Oroxylum indicum \\
\hline $\mathbf{3 .}$ & Gambhari & Gmelina arborea \\
\hline $\mathbf{4 .}$ & Patla & Stereospermum sauveolens \\
\hline $\mathbf{5 .}$ & Agnimantha & Premna obtusifolia \\
\hline $\mathbf{6 .}$ & Shlaparni & Desmodium gengeticum \\
\hline $\mathbf{7 .}$ & Prishnaparni & Urariapicta \\
\hline $\mathbf{8 .}$ & Gokshur & Tribulus terrestris \\
\hline $\mathbf{9 .}$ & Brihati & Solanum indicum \\
\hline $\mathbf{1 0}$ & Kantkari & Solanum xanthocarpum \\
\hline & Kalka Dravya & Botanical Name \\
\hline $\mathbf{1 .}$ & Shatapushpa & Acorus calamus \\
\hline $\mathbf{2 .}$ & Kushtha & Saussurea lappa \\
\hline $\mathbf{3 .}$ & Bala & Sida cordifolia \\
\hline $\mathbf{4 .}$ & Guduchi & Tinospora cordifolia \\
\hline $\mathbf{5 .}$ & Ajwain & Trachyspermum \\
\hline $\mathbf{6 .}$ & Madanfala & Randia spinosa \\
\hline $\mathbf{7 .}$ & Bela & Jasminum sambac \\
\hline $\mathbf{8 .}$ & Nagarmustak & Cyperus rotundus \\
\hline $\mathbf{9 .}$ & Pippali & Piper longum \\
\hline $\mathbf{1 0}$ & Vacha & Acorus calamus \\
\hline $\mathbf{1 1}$ & *Broth of goat flesh & - \\
\hline
\end{tabular}

*some patients were not willing for using goat flesh, so it had to be excluded in Kalka Dravya of basti.

Table 3

\begin{tabular}{|l|l|l|}
\hline S.No. & Additive of Basti & Quantity \\
\hline 1. & Kwatha & $200 \mathrm{ml}$ \\
\hline 2. & Kalka & $10 \mathrm{gm}$ \\
\hline 3. & Kanji & $30 \mathrm{ml}$ \\
\hline 4. & Madhu (honey) & $50 \mathrm{ml}$ \\
\hline 5. & Saindhavalavana (rock salt) & $10 \mathrm{gm}$ \\
\hline 6. & Tila tail (sesame oil) & $50 \mathrm{ml}$ \\
\hline
\end{tabular}

Table 4: Kaal Basti shchedule ${ }^{11}$

\begin{tabular}{|l|l|l|l|}
\hline S. No. & Day & Basti & Dose \\
\hline 1. & $1^{\text {st }}$ & A & $75 \mathrm{ml}$ \\
\hline 2. & $2^{\text {nd }}$ & A & $75 \mathrm{ml}$ \\
\hline 3. & $3^{\text {rd }}$ & N & $350 \mathrm{ml}$ \\
\hline 4. & $4^{\text {th }}$ & A & $75 \mathrm{ml}$ \\
\hline 5. & $5^{\text {th }}$ & N & $350 \mathrm{ml}$ \\
\hline 6. & $6^{\text {th }}$ & A & $75 \mathrm{ml}$ \\
\hline 7. & $7^{\text {th }}$ & N & $350 \mathrm{ml}$ \\
\hline 8. & $8^{\text {th }}$ & A & $75 \mathrm{ml}$ \\
\hline 9. & $9^{\text {th }}$ & N & $350 \mathrm{ml}$ \\
\hline
\end{tabular}




\begin{tabular}{|l|l|l|l|}
\hline 10. & $10^{\text {th }}$ & A & $75 \mathrm{ml}$ \\
\hline 11. & $11^{\text {th }}$ & N & $350 \mathrm{ml}$ \\
\hline 12. & $12^{\text {th }}$ & A & $75 \mathrm{ml}$ \\
\hline 13. & $13^{\text {th }}$ & N & $350 \mathrm{ml}$ \\
\hline 14. & $14^{\text {th }}$ & A & $75 \mathrm{ml}$ \\
\hline 15. & $15^{\text {th }}$ & A & $75 \mathrm{ml}$ \\
\hline 16. & $16^{\text {th }}$ & A & $75 \mathrm{ml}$ \\
\hline
\end{tabular}

\section{A- Anuvasanabasti N-Niruhabasti}

\section{Criteria for Inclusion (CCRAS Protocol)}

- Age -20 to 70 years

- Sex - either sex

- Duration of illness - up to 2 years

- Radiating pain, starting from the gluteal region towards, the foot

- Tenderness of the sciatic nerve course

- Severe pain on squatting

- Sensory change

- Non-involvement of urinary bladder and rectum

- Positive straight leg raising sign

Criteria for Exclusion (CCRAS Protocol)

- Age below 20 years and above 70 years

- Duration of disease more than 2 years
- Monoplegia

- Paraplegia

- Hip joint arthritis

- T.B. Spine/Hip

- Pelvic pathology

- Traumatic lesion in lumbo-sacral region

Assessment Criteria (CCRAS Protocol)

- Each sign and symptom is graded, and a numerical value is given for assessment of results. The change and relief in symptoms were observed on completion of treatment.

- Overall effect of the therapy will be graded as follows:

\begin{tabular}{|l|l|}
\hline Complete remission & $100 \%$ relief \\
\hline Marked improvement & $75 \%$ to $<100 \%$ relief \\
\hline Moderate improvement & $50 \%$ to $<75 \%$ relief \\
\hline Mild improvement & $25 \%$ to $<50 \%$ relief \\
\hline No improvement & $<25 \%$ relief \\
\hline - Clinical parameters: (CCRAS Protocol) $)^{\mathbf{1 2}}$ & \\
\hline
\end{tabular}

Table 5

\begin{tabular}{|c|c|c|c|}
\hline S.No. & Subjective Symptoms & Parameters & Scoring \\
\hline \multirow[t]{4}{*}{1.} & Pricking pain (Toda) & Absent & 0 \\
\hline & & Mild & 1 \\
\hline & & Moderate & 2 \\
\hline & & Severe & 3 \\
\hline \multirow[t]{4}{*}{2.} & Pulling pain $(R u k)$ & Absent & 0 \\
\hline & & Mild & 1 \\
\hline & & Moderate & 2 \\
\hline & & Severe & 3 \\
\hline \multirow[t]{4}{*}{3.} & Stiffness (Stambha) & Absent & 0 \\
\hline & & Mild & 1 \\
\hline & & Moderate & 2 \\
\hline & & Severe & 3 \\
\hline
\end{tabular}




\begin{tabular}{|c|c|c|c|}
\hline \multirow[t]{4}{*}{4.} & Numbness (Supti) & Absent & 0 \\
\hline & & Mild & 1 \\
\hline & & Moderate & 2 \\
\hline & & Severe & 3 \\
\hline \multirow[t]{7}{*}{5.} & Twitching (Spandan) & No twitching & 0 \\
\hline & & Sometimes for $5-10 \mathrm{~min}$ & 1 \\
\hline & & Daily for 5-10 min & 2 \\
\hline & & Daily for $10-30 \mathrm{~min}$ & 3 \\
\hline & & Daily for $30-60 \mathrm{~min}$ & 4 \\
\hline & & Daily more than 1 hour & 5 \\
\hline & Subjective Signs & & \\
\hline \multirow[t]{4}{*}{6.} & Tenderness of the sciatic & No tenderness & 0 \\
\hline & Nerve & Patient says there is tenderness & 1 \\
\hline & & Patient winces & 2 \\
\hline & & Winces \& withdraws the affected limb & 3 \\
\hline \multirow[t]{4}{*}{7.} & Sensory impairment & Absent & 0 \\
\hline & & Mild & 1 \\
\hline & & Moderate & 2 \\
\hline & & Severe & 3 \\
\hline \multirow[t]{4}{*}{8.} & Walking speed & Up to 20 seconds & 0 \\
\hline & & 21 to 40 seconds & 1 \\
\hline & & 41 to 60 seconds & 2 \\
\hline & & Above 60 seconds & 3 \\
\hline \multirow[t]{9}{*}{9.} & Posture & No complaint & 0 \\
\hline & & $\begin{array}{l}\text { Patient can walk without difficulty but experienced difficulty from getting } \\
\text { up from squatting posture }\end{array}$ & 1 \\
\hline & & Difficulty to squat & 2 \\
\hline & & Difficulty in climbing upstairs & 3 \\
\hline & & Limping gait & 4 \\
\hline & & Can stand on both limbs but with pain & 5 \\
\hline & & Can stand without touching the affected limb on the floor & 6 \\
\hline & & Can sit on bed without support but with pain and difficulty & 7 \\
\hline & & Lying in bed with pain affected limb flexed by a supportive pillow & 8 \\
\hline S.No. & Objective Sign & Parameters & Scoring \\
\hline \multirow[t]{10}{*}{10.} & Straight leg raising test & 0 & 54 \\
\hline & (in degrees) & 10 & 48 \\
\hline & & 20 & 42 \\
\hline & & 30 & 36 \\
\hline & & 40 & 30 \\
\hline & & 50 & 24 \\
\hline & & 60 & 18 \\
\hline & & 70 & 12 \\
\hline & & 80 & 6 \\
\hline & & 90 & 0 \\
\hline
\end{tabular}


Table 6

\begin{tabular}{|c|c|c|c|c|c|c|c|c|c|c|c|c|c|c|c|c|c|c|c|c|c|}
\hline \multirow[t]{2}{*}{$\begin{array}{l}\text { Pa- } \\
\text { tient }\end{array}$} & \multirow[t]{2}{*}{$\begin{array}{l}\text { Se } \\
x\end{array}$} & \multicolumn{2}{|c|}{$\begin{array}{l}\text { Prick- } \\
\text { ing } \\
\text { pain }\end{array}$} & \multicolumn{2}{|c|}{$\begin{array}{l}\text { Pulling } \\
\text { pain }\end{array}$} & \multicolumn{2}{|c|}{$\begin{array}{l}\text { Stiff- } \\
\text { ness }\end{array}$} & \multicolumn{2}{|c|}{$\begin{array}{l}\text { Numb- } \\
\text { ness }\end{array}$} & \multicolumn{2}{|c|}{$\begin{array}{l}\text { Twitch- } \\
\text { ing }\end{array}$} & \multicolumn{2}{|c|}{$\begin{array}{l}\text { Tender- } \\
\text { ness of } \\
\text { the sciatic } \\
\text { nerve }\end{array}$} & \multicolumn{2}{|c|}{$\begin{array}{l}\text { Sensory } \\
\text { impair- } \\
\text { ment }\end{array}$} & \multicolumn{2}{|c|}{$\begin{array}{l}\text { Walk- } \\
\text { ing } \\
\text { speed }\end{array}$} & \multirow{2}{*}{$\begin{array}{l}\text { Pos- } \\
\text { ture }\end{array}$} & \multicolumn{3}{|c|}{$\begin{array}{l}\text { SLR Test } \\
\text { (score) } \\
\text { (effected leg) }\end{array}$} \\
\hline & & $\begin{array}{l}\mathrm{B} \\
\mathrm{T}\end{array}$ & $\begin{array}{l}\mathrm{A} \\
\mathrm{T}\end{array}$ & $\begin{array}{l}\mathrm{B} \\
\mathrm{T}\end{array}$ & $\begin{array}{l}\mathrm{A} \\
\mathrm{T}\end{array}$ & $\begin{array}{l}\mathrm{B} \\
\mathrm{T}\end{array}$ & $\begin{array}{l}\mathrm{A} \\
\mathrm{T}\end{array}$ & BT & $\mathrm{AT}$ & BT & $\begin{array}{l}\mathrm{A} \\
\mathrm{T}\end{array}$ & BT & AT & BT & AT & $\begin{array}{l}\mathrm{B} \\
\mathrm{T}\end{array}$ & $\begin{array}{l}\text { A } \\
T\end{array}$ & & $\begin{array}{l}\mathrm{A} \\
\mathrm{T}\end{array}$ & $\begin{array}{l}\mathrm{B} \\
\mathrm{T}\end{array}$ & $\begin{array}{l}\mathrm{A} \\
\mathrm{T}\end{array}$ \\
\hline 1. & $\mathrm{M}$ & 3 & 1 & 2 & 0 & 3 & 0 & 3 & 0 & 5 & 1 & 3 & 1 & 3 & 1 & 3 & 0 & 7 & 3 & 42 & 24 \\
\hline 2. & $\mathrm{M}$ & 3 & 0 & 3 & 1 & 2 & 0 & 3 & 1 & 4 & 0 & 0 & 0 & 0 & 0 & 3 & 0 & 5 & 1 & 36 & 12 \\
\hline 3. & $\mathrm{M}$ & 2 & 0 & 2 & 0 & 3 & 0 & 2 & 0 & 4 & 1 & 1 & 0 & 3 & 0 & 3 & 1 & 6 & 1 & 30 & 6 \\
\hline 4. & $\mathrm{M}$ & 3 & 1 & 3 & 1 & 0 & 0 & 3 & 1 & 4 & 0 & 1 & 0 & 2 & 1 & 2 & 0 & 6 & 1 & 42 & 12 \\
\hline 5. & $\mathrm{M}$ & 3 & 1 & 0 & 0 & 0 & 0 & 2 & 1 & 3 & 0 & 2 & 0 & 1 & 0 & 3 & 0 & 5 & 2 & 30 & 18 \\
\hline 6. & $\mathrm{~F}$ & 3 & 0 & 2 & 0 & 2 & 0 & 1 & 0 & 5 & 1 & 3 & 1 & 2 & 0 & 2 & 1 & 7 & 1 & 42 & 24 \\
\hline 7. & $\mathrm{M}$ & 2 & 0 & 0 & 0 & 3 & 1 & 0 & 0 & 4 & 1 & 2 & 0 & 2 & 1 & 1 & 0 & 4 & 1 & 36 & 12 \\
\hline 8. & $\mathrm{M}$ & 0 & 0 & 3 & 1 & 0 & 0 & 3 & 1 & 4 & 1 & 2 & 0 & 3 & 1 & 2 & 0 & 4 & 1 & 36 & 12 \\
\hline 9. & $\mathrm{M}$ & 2 & 1 & 2 & 0 & 1 & 0 & 3 & 1 & 4 & 0 & 0 & 0 & 0 & 0 & 1 & 0 & 5 & 2 & 30 & 6 \\
\hline 10. & $\mathrm{~F}$ & 3 & 0 & 0 & 0 & 3 & 0 & 2 & 0 & 3 & 0 & 3 & 1 & 1 & 0 & 3 & 0 & 5 & 1 & 24 & 6 \\
\hline
\end{tabular}

Statistical Analysis: Statistical analysis was carried out by using Wilcoxon Sign Rank Test for subjective parameters and Paired t-Test for objective parameter.
The result was interpreted at $\mathrm{P}<0.05, \mathrm{P}<0.01, \mathrm{P}<0.001$ as level of significance.

\section{Result}

Table 7

\begin{tabular}{|c|c|c|c|c|c|c|c|c|c|c|}
\hline \multirow{2}{*}{$\begin{array}{l}\text { Subjective parame- } \\
\text { ters }\end{array}$} & \multirow[t]{2}{*}{$\mathbf{W} / \mathbf{T}+$} & \multirow[t]{2}{*}{ T- } & \multicolumn{2}{|l|}{ Mean } & \multirow[t]{2}{*}{ MD } & \multirow[t]{2}{*}{$\%$ Relief } & \multirow[t]{2}{*}{ SD } & \multirow[t]{2}{*}{ SE } & \multirow[t]{2}{*}{ P-value } & \multirow[t]{2}{*}{ Significance } \\
\hline & & & BT & AT & & & & & & \\
\hline Pricking pain & 45 & 0 & 2.400 & 0.400 & 2.0 & $83.33 \%$ & 0.9428 & 0.2981 & 0.0039 & Very significant \\
\hline Pulling pain & 28 & 0 & 1.700 & 0.300 & 1.4 & $82.35 \%$ & 0.9661 & 0.3055 & 0.0156 & Significant \\
\hline Stiffness & 28 & 0 & 1.700 & 0.100 & 1.6 & $94.11 \%$ & 1.265 & 0.4000 & 0.0078 & Very significant \\
\hline Numbness & 45 & 0 & 2.200 & 0.500 & 1.7 & $77.27 \%$ & 0.8233 & 0.2603 & 0.0039 & Very significant \\
\hline Twitching & 55 & 0 & 4 & 0.500 & 3.5 & $87.50 \%$ & 0.5270 & 0.1667 & 0.0020 & Very significant \\
\hline $\begin{array}{l}\text { Tenderness of the } \\
\text { sciatic nerve }\end{array}$ & 36 & 0 & 1.700 & 0.300 & 1.4 & $82.35 \%$ & 0.8433 & 0.2667 & 0.0039 & Very significant \\
\hline Sensory impairment & 36 & 0 & 1.700 & 0.400 & 1.3 & $76.47 \%$ & 0.9487 & 0.3000 & 0.0078 & Very significant \\
\hline Walking speed & 55 & 0 & 2.300 & 0.200 & 2.1 & $91.30 \%$ & 0.8756 & 0.2769 & 0.0020 & Very significant \\
\hline Posture & 55 & 0 & 5.4 & 1.400 & 4.0 & $74.47 \%$ & 1.054 & 0.3333 & 0.0020 & Very significant \\
\hline Objective parameter & $\begin{array}{l}\text { t val- } \\
\text { ue }\end{array}$ & & & & & & & & & \\
\hline $\begin{array}{l}\text { SLR Test (effected } \\
\text { leg) }\end{array}$ & 13.500 & - & 34.800 & 13.200 & 21.60 & $62.06 \%$ & 5.060 & 1.600 & $<0.0001$ & $\begin{array}{l}\text { Extremely sig- } \\
\text { nificant }\end{array}$ \\
\hline
\end{tabular}

Table 8

\begin{tabular}{|c|c|c|c|c|c|c|c|c|c|c|c|c|}
\hline \multicolumn{3}{|c|}{ Patient } & \multirow[t]{2}{*}{$\mathbf{W} / \mathbf{T}+$} & \multirow[t]{2}{*}{ T- } & \multicolumn{2}{|c|}{ Mean } & \multirow[t]{2}{*}{ MD } & \multirow[t]{2}{*}{ \%Relief } & \multirow[t]{2}{*}{ SD } & \multirow[t]{2}{*}{ SE } & \multirow[t]{2}{*}{ P-value } & \multirow[t]{2}{*}{ significance } \\
\hline S.NO. & Age(in year) & Sex & & & BT & AT & & & & & & \\
\hline 1. & 30 & $\mathrm{M}$ & 55 & 0 & 7.40 & 3.10 & 4.30 & $58.10 \%$ & 4.87 & 1.54 & 0.0020 & Very significant \\
\hline 2. & 40 & $\mathrm{M}$ & 36 & 0 & 5.90 & 1.50 & 4.40 & $74.24 \%$ & 7.02 & 2.22 & 0.0078 & Very significant \\
\hline 3. & 66 & $\mathrm{M}$ & 55 & 0 & 5.60 & 0.90 & 4.70 & $83.92 \%$ & 6.86 & 2.17 & 0.0020 & Very significant \\
\hline 4. & 35 & $\mathrm{M}$ & 45 & 0 & 6.60 & 1.70 & 4.90 & $74.24 \%$ & 8.93 & 2.82 & 0.0039 & Very significant \\
\hline
\end{tabular}




\begin{tabular}{|l|l|l|l|l|l|l|l|l|l|l|l|l|}
\hline 5. & 31 & $\mathrm{M}$ & 36 & 0 & 4.90 & 2.20 & 2.70 & $\mathbf{5 5 . 1 0 \%}$ & 3.46 & 1.09 & 0.0078 & Very significant \\
\hline 6. & 39 & $\mathrm{~F}$ & 55 & 0 & 6.90 & 2.80 & 4.10 & $\mathbf{5 9 . 4 2 \%}$ & 5.10 & 1.61 & 0.0020 & Very significant \\
\hline 7. & 40 & $\mathrm{M}$ & 36 & 0 & 5.40 & 1.60 & 3.80 & $\mathbf{7 0 . 3 7 \%}$ & 7.17 & 2.27 & 0.0078 & Very significant \\
\hline 8. & 51 & $\mathrm{M}$ & 36 & 0 & 5.70 & 1.70 & 4.00 & $\mathbf{7 0 . 1 7 \%}$ & 7.10 & 2.24 & 0.0078 & Very significant \\
\hline 9. & 30 & $\mathrm{M}$ & $32 / 34$ & -2 & 4.70 & 1.10 & 3.60 & $\mathbf{7 6 . 5 9 \%}$ & 7.32 & 2.31 & 0.0234 & Significant \\
\hline 10. & 30 & $\mathrm{~F}$ & 45 & 0 & 4.70 & 0.80 & 3.90 & $\mathbf{8 2 . 9 7 \%}$ & 5.08 & 1.60 & 0.0039 & Very significant \\
\hline
\end{tabular}

\begin{tabular}{|l|l|l|l|}
\hline S.No. & Overall assessment & No. of Patients & Percentage \\
\hline 1. & Complete remission & 00 & $0 \%$ \\
\hline 2. & Marked improvement & 03 & $30 \%$ \\
\hline 3. & Moderate improvement & 07 & $70 \%$ \\
\hline 4. & Minor improvement & 00 & $0 \%$ \\
\hline 5. & No improvement & 00 & $0 \%$ \\
\hline
\end{tabular}

\section{OBSERVATION}

The effect of Dashmooladi Niruha Basti and Rasaraj Rasa were observed on the clinical parameters under criteria for assessment. There was $83.33 \%$ relief in pricking pain, $82.35 \%$ relief in pulling pain, $94.11 \%$ relief in stiffness, $77.27 \%$ relief in numbness, $87.50 \%$ relief in twitching, $82.35 \%$ relief in tenderness of the sciatic nerve, $76.47 \%$ relief in sensory impairment, $91.30 \%$ relief in walking speed and $74.47 \%$ relief in posture which is statistically very significant at the level of $\mathrm{P}<0.05$. In SLR Test there was $62.06 \%$ of relief percentage which is statistically extremely significant at the level of $\mathrm{P}<0.0001 \quad(\mathrm{t}=13.50)$. The overall effect of the treatment in all patients suggested that, complete cure was not in any patient. Three patients were markedly improved, and seven patients were moderately improved. None of patients remained unchanged.

\section{DISCUSSION}

Basti therapy is advocated in the line of treatment for Gridhrasi by almost all the Acharyas. The idea behind the therapy is supposed to increase strength of the Gridhrasi Nadi, the corresponding vertebrae and other involved structures. According to Acharya Parashara Guda is the Mula of the body where all Sira are located. The drug administered through Guda reaches first to Pakvashaya and then to Grahani. ${ }^{13}$ Pakvashayais the site of Purishdhara Kala and Grihani is the site of Pittadhara Kala. So Basti directly acts on Purishdhara Kala and Pittadhara Kala. Comment or
Dalhana has commented that Purishdhara \& Asthidhara Kala and Pittadhara \& Majjadhara Kala are same (Dalhana on Su. Ka.4/40). Vata Nadi is also made by Majja Dhatu and Majja is the seat of Vata Dosha. So Basti is useful in disorders of nervous system. Dashmooladi Niruha Basti consist Dsahmool Dravya, Saindhava Lavana, Madhu and Sneha as a active ingredients. According to Acharya SushrutaDashmool Dravya are Kapha-PittaVataShamaka, Amadosha Pachaka and Sarvajwaravinashak $\mathbf{1}^{\mathbf{1 4}}$ (antipyretic) in properties. Saindhava Lavana has Sukshma and Tikshna Guna, it reaches up to the micro channels and breaks down the morbid Mala and Dosha Sanghata. The presence of $\mathrm{Na}^{+}$(Saindha$v a$ ) in Basti Dravya may play important role for the absorption of the drug as the $\mathrm{Na}^{+}$channels. The most commonly used channel for absorption of the substances. Madhu (honey) is made of various substances and considered best among the vehicles (Ch.Su.27/249). Charaka says that Basti containing excess of Madhu when administered to the person makes him extremely potent (Ch.Si.12/28). Sneha Dravya reduces Vata Dushti, softens microchannels, destroys the compact mala and removes the obstruction in the channels (Ch.Si.1/7). So Dashmooladi Niruha Basti subsided pricking and pulling pain, reduced stiffness and tenderness, also showed improvement in sensory impairment. Rasaraj Rasa contains Parada (mercury), Abhraka (mica), Swarna (gold), Roupya (silver), Loha (ferrum) and Vanga (stannum) Bhasma. Jatifala, Ksheerkakoli, Kumari and 
Kakamachi as Bhavana Dravya. Rasaraj Rasa has ability to pacify vitiated Vata Doshas, majority of the ingredients are Tikta, Madhura and Kashaya Rasa predominant with Kinchit Katu Rasa, Sheet Virya, Madhura Vipaka, Laghu, Snighdha and Vyavayi in nature with alleviating action on all Doshas. Abhraka Bhasma is hepatoprotective, Lauha Bhasma is an antioxidant and Tamra Bhasma is antibacterial in properties. Ashwagandha acts as immunomodulator and has anti-inflammatory effect. Swarna and Tamra Bhasma have free radical scavenging properties. Rasaraj Rasa reduced numbness, twitching and inflammation etc. in patients.

\section{CONCLUSION}

On the basis of this clinical trial it can be concluded that Dashmooladi Niruha Basti and Rasaraj Rasa work effectively in the management of Gridhrasi. This Ayurvedic management prevents the progression of disease and improves the quality of life of the patient. This research paper evaluated the effective management of Gridhrasi (Sciatica) through Ayurveda perspective. Though this trial was carried out on only 10 patients, large number of sample size is necessary which may be the scope of further research.

\section{REFERENCES}

1. H Ralston Stuart, D Penman Ian, et.al., Davidson's Principles and Practice of Medicine, 23rd edition 2018, Ch. 25, Page no. 1064 and 1134.

2. Kasper Dennis L., Fauci Anthony S. et. Al., Harrison's Principles of Internal Medicine, $19^{\text {th }}$ edition 2015, Mc Graw Hill Publication, Page no. 2222.

3. www.omicsonline.org $>$ india $>$ sciatica

4. H Ralston Stuart, D Penman Ian, et.al., Davidson's Principles and Practice of Medicine, 23rd edition 2018, Ch. 25, Page no. 1135.

5. Shastri Kashinath \& Chaturvedi Gourakha Nath, edited Charak Samhita of Agnivesh, revised by Charak and Dridhbala, Part-1, Chaukhambha Sanskrit Sansthan, Varanasi Reprint 2017; Sutrasthana 19, verse 3, page no. 381 .

6. Shastri Kashinath \& Chaturvedi Gourakha Nath, edited Charak Samhita of Agnivesh, revised by Charak and Dridhbala, Part-1, Chaukhambha Sanskrit Sansthan,
Varanasi Reprint 2017; Sutrasthana 20, verse 11, page no. 399.

7. Shastri Kashinath \& Chaturvedi Gourakha Nath, edited Charak Samhita of Agnivesh, revised by Charak and Dridhbala, Part-2, Chaukhambha Sanskrit Sansthan, Varanasi Reprint 2017; Chikitsasthana 28, verse 58, page no. 788 .

8. Shastri Kashinath \& Chaturvedi Gourakha Nath, edited Charak Samhita of Agnivesh, revised by Charak and Dridhbala, Part-2, Chaukhambha Sanskrit Sansthan, Varanasi Reprint 2017; Chikitsasthana 28, verse 56, page no.787.

9. Shastri Kashinath \& Chaturvedi Gourakha Nath, edited Charak Samhita of Agnivesh, revised by Charak and Dridhbala, Part-2, Chaukhambha Sanskrit Sansthan, Varanasi Reprint 2017; Siddhisthana 3, verse 35, page no. 999 .

10. Shastri Kaviraj Ambikadatta, Bhaisajyaratnavali, Vidhyotini Hindi Commentary, Chaukhamba Sanskrit Sansthan, Varanasi $16^{\text {th }}$ edition 2002, Vatavyadhi Prakaran 26, verse 204-208.

11. Shastri Kashinath \& Chaturvedi Gourakha Nath, edited Charak Samhita of Agnivesh, revised by Charak and Dridhbala, Part-2, Chaukhambha Sanskrit Sansthan, Varanasi Reprint 2017; Siddhisthana 1, verse 48, page no. 973 .

12. Clinical Research Protocol for traditional health sciences, Central Council for Research in Ayurveda \& Siddha.

13. Shastri Kashinath \& Chaturvedi Gourakha Nath, edited Charak Samhita of Agnivesh, revised by Charak and Dridhbala, Part-2, Chaukhambha Sanskrit Sansthan, Varanasi Reprint 2017; Siddhisthana 3, verse 24, page no. 994.

14. Shastri Ambikadutta, Sushruta Samhita, AyurvedaTattva- Sandipika Hindi Commentry, Part-1, Chaukhambha Sanskrit Sansthan Varanasi Reprint, 2016; Sutra Sthana 38, verse 72, page no. 189.

\section{ABBREVIATIONS}

SLR: Straight Leg Raising, MRI: Magnetic Resonance Imaging, A.H.Ni.: AshtangHridyaNidanasthana, Ch. Chi: CharakChikitsasthana, Ch. Si.: CharakSiddhiasthana, Ch. Su.: CharakSutrasthana, Ma. Ni.: Madhava Nidana, Su. Ka.: SushrutaKalpasthana, Su. Ni.: SushrutaNidanasthana, BT: Before Treatment, 


\begin{abstract}
AT: After Treatment,
MD: Mean Difference,

SD: Standard Deviation,

SE: Standard Error,

CI: Confidence Interval
\end{abstract}

\title{
Source of Support: Nil
}

\section{Conflict of Interest: None Declared}

How to cite this URL: Mishra Meenu et al: Clinical Assessment Of Basti Karma And Rasaraj Rasa In The Management Of Gridhrasi (Sciatica). International Ayurvedic Medical Journal \{online\} 2020 \{cited October, 2020\} Available from: http://www.iamj.in/posts/images/upload/4684_4693.pdf 